University of Nebraska - Lincoln

DigitalCommons@University of Nebraska - Lincoln

$11-10-2006$

\title{
High-resolution imaging of proteins in human teeth by scanning probe microscopy
}

\author{
Alexei Gruverman \\ University of Nebraska-Lincoln, agruverman2@unl.edu \\ D. $\mathrm{Wu}$ \\ North Carolina State University, Raleigh, NC \\ B. J. Rodriguez \\ Condensed Matter Sciences Division, Oak Ridge National Laboratory, brian.rodriguez@ucd.ie \\ Sergei V. Kalinin \\ Oak Ridge National Laboratory, sergei2@ornl.gov \\ S. Habelitz \\ University of California, San Francisco, CA
}

Follow this and additional works at: https://digitalcommons.unl.edu/physicsgruverman

Part of the Physics Commons

Gruverman, Alexei; Wu, D.; Rodriguez, B. J.; Kalinin, Sergei V.; and Habelitz, S., "High-resolution imaging of proteins in human teeth by scanning probe microscopy" (2006). Alexei Gruverman Publications. 24.

https://digitalcommons.unl.edu/physicsgruverman/24

This Article is brought to you for free and open access by the Research Papers in Physics and Astronomy at DigitalCommons@University of Nebraska - Lincoln. It has been accepted for inclusion in Alexei Gruverman Publications by an authorized administrator of DigitalCommons@University of Nebraska - Lincoln. 


\title{
High-resolution imaging of proteins in human teeth by scanning probe microscopy
}

\author{
A. Gruverman ${ }^{\mathrm{a}, *}$, D. Wu ${ }^{\text {a }}$, B.J. Rodriguez ${ }^{\mathrm{b}}$, S.V. Kalinin ${ }^{\mathrm{b}}$, S. Habelitz ${ }^{\mathrm{c}}$ \\ ${ }^{a}$ Departments of Materials Science and Engineering and Physics, North Carolina State University, Raleigh, NC 27695, USA \\ ${ }^{\mathrm{b}}$ Condensed Matter Sciences Division, Oak Ridge National Laboratory, Oak Ridge, TN 37831, USA \\ ${ }^{c}$ Department of Preventive and Restorative Dental Sciences, University of California, San Francisco, CA 94143, USA
}

Received 24 October 2006

Available online 10 November 2006

\begin{abstract}
High-resolution studies of dental tissues are of considerable interest for biomedical engineering and clinical applications. In this paper, we demonstrate the application of piezoresponse force microscopy (PFM) to nanoscale imaging of internal structure of human teeth by monitoring the local mechanical response to an electrical bias applied via a conductive tip. It is shown that PFM is capable of detecting dissimilar components of dental tissues, namely, proteins and calcified matrix, which have resembling morphology but different piezoelectric properties. It is demonstrated that collagen fibrils revealed in chemically treated intertubular dentin exhibit high piezoelectric activity and can be visualized in PFM with spatial resolution of $10 \mathrm{~nm}$. Evidence of the presence of protein inclusions of 100-200 nm wide and several micrometers long in tooth enamel has been obtained. Furthermore, it is found that the peritubular dentin and intertubular dentin exhibit different piezoelectric behavior suggesting different concentration of collagen fibrils. The obtained results demonstrate a high potential of PFM in providing an additional insight into the structure of dental tissues. It is suggested that the PFM approach can be used to study the structure of a wide range of biological materials by monitoring their electromechanical behavior at the nanoscale.
\end{abstract}

(c) 2006 Elsevier Inc. All rights reserved.

Keywords: Scanning probe microscopy; Piezoelectricity; Collagen, Teeth; Dentin; Biological imaging

Local mechanical and biological functionality of teeth and its relationship with local nano- and meso-structure is a matter of considerable interest for biomedical engineering and clinical applications. Information on these properties can lead to better understanding of tissue growth, development and remodeling and to delineation of interaction mechanisms between artificial and natural tissues. High-resolution characterization of dental tissues may open more possibilities for detection of the early stages of diseases in mineralized tissues and for development of better restorative materials. In the last decades, a number of approaches based on small angle X-ray diffraction [1-3], second harmonic generation microscopy [4,5], confocal

\footnotetext{
* Corresponding author.

E-mail address: alexei_gruverman@ncsu.edu (A. Gruverman).
}

microscopy [6-8], and X-ray diffraction $[9,10]$ have been developed to access the structure of calcified tissues. In parallel, high-resolution electron microscopy has been broadly used to study the structure of these materials on the nanoscale [11].

An alternative approach for local studies of biological systems is based on scanning probe microscopy (SPM). A number of atomic force microscopy (AFM) studies have addressed surface morphology of bones, teeth and other connective tissues [12-16]. Several research groups have attempted mapping the effective mechanical properties of dental tissues by means of nanoindentation $[17,18]$ and dynamic stiffness mapping [19]. These techniques allow direct nanoscale mechanical characterization of local defects as micro-cracks, variations in mineral density and caries formation sites. High-resolution imaging of 
mineralized tissue components by atomic force microscopy usually requires the modification of the surface by chemical or biochemical treatments. Collagen fibrils cannot be revealed without acid treatment and demineralization of the sample [20]. The resolution is further greatly improved if non-collagenous proteins are removed by deproteinization with $\mathrm{NaOCl}$ or trypsin [21].

Additional possibilities for characterization of biological materials can be provided by another mode of SPM, piezoresponse force microscopy (PFM), which utilizes the piezoelectric behavior in biomaterials, or linear coupling between electrical and mechanical phenomena [22,23]. Piezoelectric behavior of biological systems results from the presence of proteins and other organic components, which exhibit piezoelectric activity [24-30]. In the last decade, PFM has demonstrated its unparalleled capabilities in testing the electromechanical behavior of inorganic piezoelectric materials, such as ferroelectrics and III-V nitrides, with nanoscale resolution [31,32]. Application of PFM to dental tissues may provide an opportunity to reveal their structural components without special surface treatments. Identification of the size and orientation of protein fibrils is essential for understanding the structure of the protein-mineral complex and its correlation with the mechanical properties of teeth at the different length scales. In this paper, we demonstrate application of PFM for nanoscale imaging of internal structure of dental tissues.

\section{Materials and methods}

Human third molars with documented history were extracted from patients of ages of between 22 and 34 years according to protocols approved by the University of California, San Francisco Committee on Human Research [21]. Teeth were sterilized by $c$-radiation and stored in deionized water at $4{ }^{\circ} \mathrm{C}$ until prepared [33]. Sagittal midcoronal sections of six teeth (thickness of $2 \mathrm{~mm}$ ) were prepared by polishing through a series of $\mathrm{SiO}_{2}$ papers and with water-based diamond paste to 0:25 $\mu \mathrm{m}$ (Buehler). Ultrasonic treatments in water for $10 \mathrm{~s}$ were used to clean the surface.

Two groups (each $n=3$ ) of specimens were investigated: (1) mechanically polished only; (2) polished and etched with $10 \mathrm{vol} \%$ citric acid $\mathrm{C}_{6} \mathrm{H}_{8} \mathrm{O}_{7}$ for $15 \mathrm{~s}$ and subsequently treated with aqueous solution of $1 \mathrm{vol} \%$ sodium hypochlorite $\left(\mathrm{NaOCl}_{\mathrm{aq}}\right)$ for up to $150 \mathrm{~s}$. The application of citric acid dissolves the mineral in calcified tissues and leaves the organic phases behind. Dentin etching is a common technique for providing a better substrate for bonding to dental adhesives [34]. Sodium hypochlorite is used as a cleansing, disinfective, nonspecific deproteinizing agent in endodontic treatments [35]. The $\mathrm{NaOCl}_{\mathrm{aq}}$ treatment was applied to non-specifically remove layers of non-collagenous and collagenous proteins. As shown elsewhere [21], this procedure removes non-collagenous proteins initially and facilitates the visualization of individual collagen fibrils by atomic force microscopy (AFM).

Dentin sections were glued to metal disks and imaged both in topographic and piezoresponse modes using a commercial scanning probe microscope (Autoprobe M5 Park Scientific Instruments). The PFM mode is based on the detection of electromechanical response of a piezoelectric sample to a modulation voltage, $V_{\mathrm{tip}}=V_{\mathrm{dc}}+V_{\mathrm{ac}} \cos \omega t$, applied between the conductive AFM tip in contact with the sample surface and a conductive substrate, as illustrated in Fig. 1. The local mechanical vibration of the sample due to the converse piezoelectric effect, $d=d_{1 \omega} \cos (\omega t+\varphi)$, is detected using a standard lock-in technique with the same tip that accurately follows the surface oscillations [31]. Amplitude of the signal, $\mathrm{PR}=d_{1 \omega} / V_{\mathrm{ac}}$, provides information on the effective piezoelectric constant

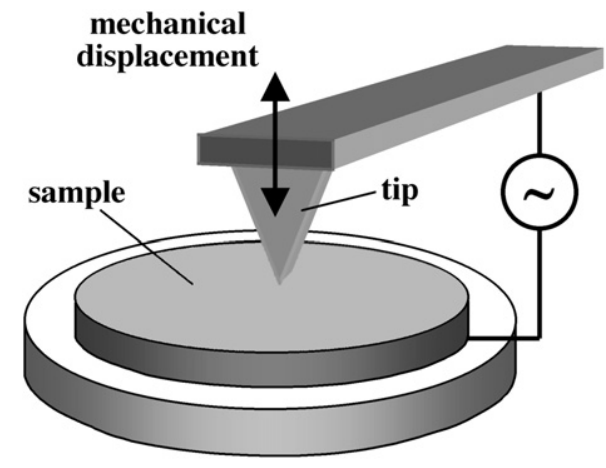

Fig. 1. PFM experimental geometry for mapping the electromechanical response. A conductive tip is used to apply an electrical bias and to detect the local electromechanical response of the sample.

while phase, $\varphi$, depends on its sign and changes between regions with different crystallographic orientation. The unique strength of PFM is that both vertical and lateral components of surface displacement can be measured [31], providing information on both normal and in-plane components of the electromechanical response vector. The size of the tipsample contact area, which can be smaller than $10 \mathrm{~nm}$ in diameter, determines the nanoscale resolution that can be achieved in PFM. In this study, measurements were performed by applying an ac modulation voltage $(2.0 \mathrm{~V}, 15 \mathrm{kHz}$ ) to the $\mathrm{Pt}$ coated tips (resonant frequency $\sim 150 \mathrm{kHz}$, spring constant $k \sim 4.5 \mathrm{~N} / \mathrm{m}$ ).

\section{Results and discussion}

A topographic image of the dentin sample treated with $\mathrm{NaOCl}_{\mathrm{aq}}$ (Fig. 2a) shows a pattern of randomly oriented collagen fibrils with distinct axial repeat structure. Topographic observation of chemically treated dentin has been previously used to measure the dimensions of the fibril features, such as fibril diameter and axial repeat distance of individual fibrils [21]. Application of PFM allowed us to get a new insight into the dentin structure. Figs. $2 \mathrm{~b}$ and $\mathrm{c}$ show the PFM amplitude and phase images, respectively, of the same area as in Fig. 2a. Piezoelectrically active regions appearing as bright elongated areas in the PFM amplitude image correlate well with the pattern of collagen fibrils exposed in the topographic image illustrating the PFM capability of detecting the collagen fibrils by monitoring the piezoresponse signal. Furthermore, from comparison of the PFM amplitude and phase images it can be seen that fibril contrast in the phase image varies from bright to dark for different fibrils. As the PFM phase imaging is sensitive to the sign of the piezoelectric coefficient it is reasonable to attribute these phase contrast variations to random orientation of the fibrils.

Piezoelectric behavior has been observed in a number of biological systems including bones, teeth, wood, proteins, DNA and polysaccharides $[24,28,36]$. In earlier experiments, it has been shown that the piezoelectric behavior of the composite biological tissues, such as bone, stems from the crystallographic structure of collagen fibers imbedded in the mineral matrix of hydroxyapatite (HAP) crystals [26,29]. While HAP belongs to the 

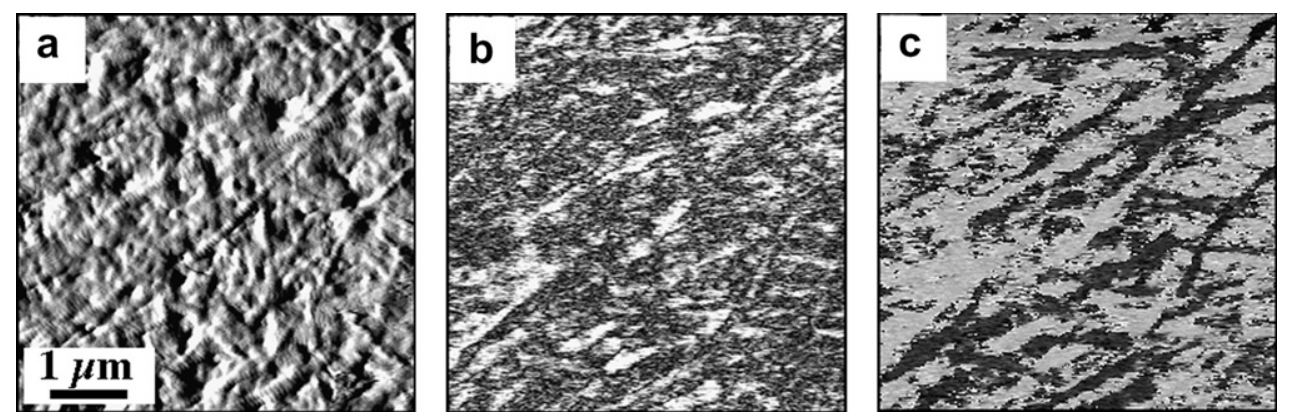

Fig. 2. Visualization of a pattern of collagen fibrils in the chemically treated dentin sample: (a) topography, (b) PFM amplitude, and (c) PFM phase. Collagen fibrils visible in the topographic image appear as elongated bright regions in the PFM amplitude image and as bright and dark regions in the PFM phase image.

centrosymmetric space group $\mathrm{P}_{3} / \mathrm{m}$ and therefore cannot exhibit piezoelectric behavior, the $D_{\infty}$ symmetry of fibrillar protein molecules results in the piezoelectric response to shear forces acting along the fiber axis and is therefore, responsible for the piezoelectric behavior of biosystems containing self-assembled proteins. Therefore, monitoring the electromechanical response (piezoresponse) allows visualization and differentiation between organic and mineral components in composite biomaterials. Results obtained in dentin samples by means of PFM illustrate that this electromechanical imaging of collagen fibers can be performed with nanoscale resolution. The origin of the directional dependence of piezoresponse may mainly be associated with the molecular orientation of tropocollagen within fibrils. Collagen fibril formation is a self-assembly process and it has been shown that tropocollagen molecules not only align themselves parallel to each other but also orient their $\mathrm{C}$ - and $\mathrm{N}$-termini in one direction. The observed differences in the piezoresponse signal of individual collagen fibrils may be associated with different or opposed orientations of the tropocollagen in one fibril compared to adjacent ones. Depending of the fibril orientation, the PFM tip will either scan in direction from C- to Nterminus or in reverse direction resulting in opposite PFM phase signals. The effect of molecular orientation on piezoelectricity is also described elsewhere [22].

During this study, it has been confirmed that PFM imaging can be effective even in the tooth samples that has not been chemically treated but only mechanically pol- ished. Fig. 3 shows the topographic and PFM images of tooth enamel in the vicinity of the dental-enamel junction (DEJ). While surface topography does not show any specific features related to dissimilar regions, PFM clearly illustrates a fibrillar structure of presumably organic nature of about 100-200 nm in width embedded within a non-piezoelectric matrix. The embedded protein fibril exhibits strong electromechanical contrast in the PFM amplitude image while there is no electromechanical activity in the surrounding mineral phase. This result seems to be consistent with the earlier studies of the tooth morphology that found that organic fibers extended from the dentin zone through DEJ into the enamel [37] to a distance of several microns. We note that these inclusions are quite common and can be also found at much larger distances from DEJ (up to $100 \mu \mathrm{m}$ ). Variations of contrast within the PFM phase image of the fibril correspond to differently oriented fibril fragments (as discussed above) presumably due to the presence of different microfibril strands. From these measurements we estimated the spatial resolution in PFM imaging, determined as a half-width of the boundary between different piezoelectric regions, to be about $5 \mathrm{~nm}$. Note again that simple morphological examination of the surface could not reveal the protein fiber in the calcified phase, which clearly illustrates the advantage of PFM in visualization of organic components in dental tissues without any special sample preparation.

Topographic image in Fig. 4a shows the surface of a mechanically polished cross-sectional dentin sample with
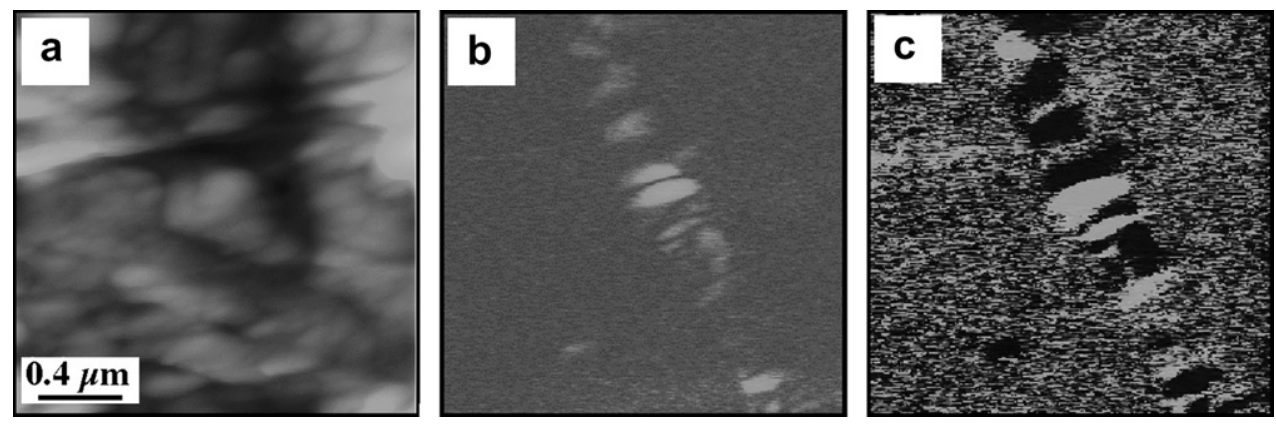

Fig. 3. High-resolution imaging of a single protein microfibril found in enamel in the vicinity of the DEJ in the mechanically polished tooth: (a) topography, (b) PFM amplitude, and (c) PFM phase. 

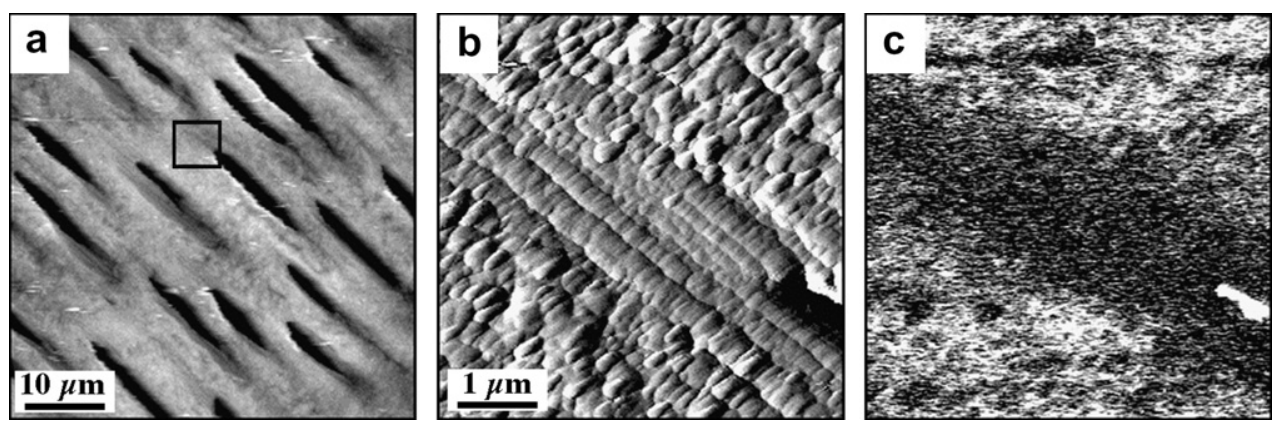

Fig. 4. (a) Topographic image of the mechanically polished cross-sectional dentin sample showing a number of dentin tubules; (b, c) zoom-in of the area marked by a block in (a): (b) topographic image of the tip of a dentin tubule, (c) PFM amplitude image of the same location showing piezoelectric activity of the intertubular dentin (bright contrast) while peritubular region does not exhibit a piezoelectric signal (dark contrast).

the characteristic tubules of about $1 \mu \mathrm{m}$ wide and $10-15 \mu \mathrm{m}$ long. Intertubular dentin (ITD) comprises the bulk of the specimen, while peritubular dentin (PTD) forms a thin $\operatorname{rim}(\sim 1 \mu \mathrm{m}$ wide $)$ that surrounds each tubule. In high-magnification image (Fig. 4b) PTD region can be identified as having slightly different morphology comprised by elongated crystallites. The PFM image (Fig. 4c) shows a moderate piezoelectric response from the intertubular dentin region which appears with brighter contrast. The effective piezoelectric coefficient determined from the measurements of the local electromechanical response as a function of the modulation bias has been estimated to be about 0.15 $0.25 \mathrm{pm} / \mathrm{V}$. No detectable piezoresponse response was obtained from the PTD region (dark contrast in PFM). Bright contrast on the tubule end at the lower right part of the image results from the oversaturated signal due to the high roughness of the surface (profile variations is of the order of $0.5 \mu \mathrm{m}$ ) and is not indicative of high piezoelectric activity. Since high piezoelectric activity in chemically treated dentin samples is associated with collagen fibrils, we attributed the lack of piezoelectricity in PTD to the absence of collagen.

Dentin originates from the expression and secretion of a primarily type-I collagenous matrix by the neural crest derived odontoblasts. This specialized cell develops a process that is several millimeters long but only $1 \mu \mathrm{m}$ in diameter. The odontoblast process embeds itself in the extracellular matrix and remains there during and after mineralization creating the characteristic tubules in dentin. PTD forms around the tubules, but the timing of its mineralization is unclear. Our studies support the hypothesis that PTD is a non-collagenous tissue and a product of the secretion of specialized proteins that facilitate the mineralization of the tubule wall. Detail studies of the ITD and PTD structures are underway and will be presented in a forthcoming paper.

\section{Summary}

To summarize, piezoresponse force microscopy is shown to be an effective tool for nanoscale imaging of electromechanically active proteins in dental tissues. PFM allows dif- ferentiation between organic and mineral components and is not sensitive to minor topographic features, thus significantly simplifying the interpretation of image contrast in terms of materials microstructure. Protein microfibrils of 100-200 $\mathrm{nm}$ wide and several micrometers long have been observed in the enamel in the vicinity of the DEJ. The obtained results demonstrate a great potential for PFM in imaging and elucidating the structure/property relationship in dental tissues. Given the fact that a number of biological systems exhibit piezoelectricity, we suggest that PFM can be used to perform studies of internal structure of the protein microfibrils in a variety of biomaterials by monitoring their electromechanical behavior.

\section{Acknowledgments}

A.G. acknowledges support of National Science Foundation (Grant No. DMR02-35632). Research was performed in part as a Eugene P. Wigner Fellow and staff member at the Oak Ridge National Laboratory (ORNL), managed by UT-Battelle, LLC, for the U.S. Department of Energy under Contract DE-AC05-00OR22725 (SVK). SH acknowledges support of NIH/NIDCR (Grant P01DE09859).

\section{References}

[1] P. Fratzl, O. Paris, K. Klaushofer, W.J. Landis, bone mineralization in an osteogenesis imperfecta mouse model studied by small-angle X-ray scattering, J. Clin. Invest. 97 (1996) 396-402.

[2] A. Ascenzi, A. Benvenuti, A. Bigi, E. Foresti, M.H.J. Koch, F. Mango, A. Ripamonti, N. Roveri, X-ray diffraction on cyclically loaded osteons, Calcif. Tissue Int. 62 (1998) 266-273.

[3] A. Bigi, A. Cacchioli, A.M. Fichera, C. Gabbi, M.H.J. Koch, L. Ragionieri, A. Ripamonti, N. Roveri, X-ray diffraction and polarizing optical microscopy investigation of the structural organization of rabbit tibia, J. Biomed. Mater. Res. 41 (1998) 289-295.

[4] A. Zoumi, A. Yeh, B.J. Tromberg, Imaging cells and extracellular matrix in vivo by using second-harmonic generation and two-photon excited fluorescence, Proc Natl. Acad. Sci. USA 99 (2002) 11014 11019.

[5] T. Yasui, Y. Tohno, T. Araki, Determination of collagen fiber orientation in human tissue by use of polarization measurement of molecular second-harmonic-generation light, Appl. Opt. 43 (2004) 2861-2867. 
[6] M. Fontana, T.L. Buller, A.J. Dunipace, G.K. Stookey, R.L. Gregory, An in vitro microbial-caries model used to study the efficacy of antibodies to streptococcus mutans surface proteins in preventing dental caries, Clin. Diagn. Lab. Immunol. 7 (2000) 49-54.

[7] M.A. Chellaiah, N. Kizer, R. Biswas, U. Alvarez, J. StraussSchoenberger, L. Rifas, S.R. Rittling, D.T. Denhardt, K.A. Hruska, Osteopontin deficiency produces osteoclast dysfunction due to reduced cd44 surface expression, Mol. Biol. Cell. 14 (2003) 173-189.

[8] J. Wu, B. Rajwa, D.L. Filmer, C.M. Hoffman, B. Yuan, C. Chiang, J. Sturgid, J.P. Robinson, Automated quantification and reconstruction of collagen matrix from 3D confocal datasets, J. Microsc. 210 (2003) $158-165$.

[9] C. Boote, S. Dennis, K. Meek, Spatial mapping of collagen fibril organisation in primate cornea - an X-ray diffraction investigation, J. Struct. Biol. 146 (2004) 359-367.

[10] A. Bigi, M. Burghammer, R. Falconi, M.H.J. Koch, S. Panzavolta, C. Riekel, Twisted plywood pattern of collagen fibrils in teleost scales: an X-ray diffraction investigation, J. Struct. Biol. 136 (2001) 137-143.

[11] W.J. Landis, K.J. Hodgens, J. Arena, M.J. Song, B.F. McEwen, The structural relation between collagen and mineral in bone as determined by high voltage electron microscopic tomography, Micros. Res. Tech. 33 (1996) 192-202.

[12] E.A.G. Chernoff, D.A. Chernoff, Atomic force microscope images of collagen fibers, J. Vac. Sci. Technol. A 10 (1992) 596-599.

[13] F. El Feninat, T.H. Ellis, E. Sacher, I. Stangel, A tapping mode AFM study of collapse and denaturation in dentinal collagen, Dent. Mater. 17 (2001) 284-288.

[14] H.G. Hansma, Surface biology of DNA by atomic force microscopy, Annu. Rev. Phys. Chem. 52 (2001) 71-92.

[15] W.J. Landis, J. Moradian-Oldak, S. Weiner, Topographic imaging of mineral and collagen in the calcifying turkey tendon, Connect. Tissue Res. 25 (1991) 181-196.

[16] G.W. Marshall, M. Balooch, R.J. Tench, J.H. Kinney, S.J. Marshall, Atomic force microscopy of acid effects on dentin, Dent. Mater. 9 (1993) 265-268.

[17] J.Y. Rho, T.Y. Tsui, G.M. Pharr, Elastic properties of human cortical and trabecular bone measured by nanoindentation, Biomaterials 18 (1997) 1325-1330.

[18] M. Balooch, I.C. Wu-Magidi, A. Balazs, A.S. Lundkvist, S.J. Marshall, G.W. Marshall, W.J. Siekhaus, J.H. Kinney, Viscoelastic properties of demineralized human dentin measured in water with atomic force microscope (AFM)-based indentation, J. Biomed. Mater. Res. 40 (1998) 539-544.

[19] G. Balooch, G.W. Marshall, S.J. Marshall, O.L. Warren, S.A.S. Asif, M. Balooch, Evaluation of a new modulus mapping technique to investigate microstructural features of human teeth, J. Biomech. 37 (2004) 1223-1232.
[20] T. Hassenkam, G.E. Fantner, J.A. Cutroni, J.C. Weaver, D.E. Morse, High-resolution AFM imaging of intact and fractured trabecular bone, Bone 35 (2004) 4-10.

[21] S. Habelitz, M. Balooch, S.J. Marshall, G. Balooch, G.W. Marshall, In situ atomic force microscopy of partially demineralized human dentin collagen fibrils, J. Struct. Biol. 138 (2002) 227-236.

[22] S.V. Kalinin, B.J. Rodriguez, S. Jesse, T. Thundat, A. Gruverman, Electromechanical imaging of biological systems with sub- $10 \mathrm{~nm}$ resolution, Appl. Phys. Lett. 87 (2005) 053901-053903.

[23] C. Halperin, S. Mutchnik, A. Argonin, M. Molotskii, P. Urenski, M. Salai, G. Rosenman, Piezoelectric effect in human bones studied in nanometer scale, NanoLetters 4 (2004) 1253-1256.

[24] E. Fukada, I. Yasuda, On the piezoelectric effect of bone, J. Phys. Soc. Jpn. 12 (1957) 1158-1162;

I. Yasuda, On the piezoelectric activity of bone, J. Jpn. Orthop. Surg. Soc. 28 (1957) 267-269.

[25] J.C. Anderson, C. Eriksson, Piezoelectric properties of dry and wet bone, Nature 227 (1970) 491-492.

[26] S.B. Lang, Pyroelectric effect in bone and tendon, Nature 5063 (1966) 704-705.

[27] E. Fukada, Piezoelectricity of wood, J. Phys. Jpn. 10 (1955) 149-154.

[28] V.A. Bazhenov, Piezoelectric properties of wood, Consultants Bureau, New York, 1961.

[29] E. Fukada, Piezoelectricity of biopolymers, Biorheology 32 (1995) 593-609.

[30] A.A. Marino, B.D. Gross, Piezoelectricity in cementum, dentine and bone, Archs. Oral Biol. 34 (1989) 507-509.

[31] M. Alexe, A. Gruverman, Nanoscale characterisation of ferroelectrics: Scanning Probe Microscopy approach, Springer Verlag, New York, 2004.

[32] B.J. Rodriguez, W.-C. Yang, R.J. Nemanich, A. Gruverman, Scanning probe investigation of surface charge and surface potential of GaN-based heterostructures, Appl. Phys. Lett. 86 (2005) 112115 112117.

[33] J.M. White, H.E. Goodis, S.J. Marshall, G.W. Marshall, Sterilization of teeth by gamma radiation, J. Dent. Res. 73 (1994) 1560 1567.

[34] G.W. Marshall, S.J. Marshall, J.H. Kinney, M. Balooch, The dentin substrate:structure and properties related to bonding, J. Dent. 25 (1997) 441-458.

[35] G.W. Marshall, N. Yucel, M. Balooch, J.H. Kinney, S. Habelitz, S.J. Marshall, Sodium hypocloride alterations of dentin collagen, Surf. Sci. 491 (2001) 444-455.

[36] M.A. El Messiery, Physical basis for piezoelectricity of bone matrix, IEE Proc. 128A (1980) 336-346.

[37] S. Habelitz, G.W. Marshall, M. Balooch, S.J. Marshall, Nanoindentation and storage of teeth, J. Biomech. 35 (2002) 995-998. 